\title{
REVIEWS
}

\section{AUTOMATIC GENERATION AND EVALUATION OF RECOMBINATION GAMES}

\author{
Doctoral Dissertation \\ by Cameron Browne \\ 2008, Queensland University of Technology \\ Email: Cameron.browne @ btinternet.com
}

Reviewed by Ingo Althöfer ${ }^{1}$

During all the decades of computer chess we had one big goal: to make computers play games as strong as possible! When our horizon broadened, this goal remained the same: to make computers play games as strong as possible: in Checkers, Hex, Go, Backgammon, Awari, Bridge, ... . For the programmers this typically meant: Concentrate on "your" specific game and try to exploit every single aspect you can identify.

A rather different goal has become of interest only recently: the computer shall be used in the process of "designing new games". Therefore the computer shall play "all" games within a large class reasonably well without tuning.

Some years ago, your reviewer used simple tree searches (rudimentary alpha-beta with straightforward evaluations and pure Monte-Carlo sampling, directly applied to root positions) to design board games with computer help (Althöfer, 2003). The task of the computer program (called a bot) was to perform long series of quick test games for "candidate rule sets" and to provide statistics on a few criteria such as game lengths, drawing quota, balance of chances, and variability. For this purpose, the bot did not need to be a strong player. Instead, it was much more important that a series of say 1,000 test games could be executed within a few seconds or at most minutes on a normal PC. I really liked the staccato of modifying rules - selfplay series modifying rules - selfplay series - modifying rules - ..., with dozens of generations in a single afternoon or evening session.

Cameron Browne (2008a) went a large step further. His goal was not computer-aided game invention but fully automatic game invention. Browne created a list of 57 aesthetic criteria for a board game. Then he asked 106 human individuals (from all over the world) to identify the best 17 criteria in this list, by applying them to some 79 known games. These 17 top criteria were used by Browne's program LUDI (based on a genetic algorithm) to evaluate games, with the help of long series of selfplay.

Here, we discuss only one of the 17 criteria: Drama, as it is called by Browne. He states "Drama is the tendency for players to recover from seemingly bad positions. Drama is good for a game as it means that the leading player will not necessarily win the game and the eventual outcome remains uncertain until that lead becomes decisive." For a proper understanding of the background of game tree search history we should know that Browne's LUDI did not use Monte-Carlo techniques, but only traditional alpha-beta search with iterative deepening.

During the last two weeks of the Ph.D. project LUDI was engaged in a very long run: the computer started with a population of 79 known games. During the process altogether 1,389 new games were created by repeated recombination, mutation, and selection. At the end, the top 19 new games were ranked - and for reasons of soundness became also evaluated by 27 human test players. Some of these games are really nice. My favourite is Yavalath, on rank four in the bot's aesthetical list, and on rank two in the opinion of the 27 individuals. Yavalath is a " 4 in a row" game but with the additional trick that " 3 in a row without simultaneously 4 in a row" is a loss.

\footnotetext{
${ }^{1}$ Faculty of Mathematics and Computer Science, Friedrich-Schiller University Jena, 07737 Jena, Germany. Email: ingo.althoefer@uni-jena.de
} 


\section{Yavalath: The formal rules}

Players: White and Black. Each player has 30 pieces of his colour.

Start: The board has a hexagonal shape and consists of 61 hexagonal squares (so side length is 5). Initially the board is empty.

Play: Players take turns placing a piece of their colour in an empty cell.

End: Players win by making a line of four pieces of their colour and lose by making a line of three pieces of their colour without also making a line of four. The game is drawn if the board fills up before either player wins or loses.

Yavalath (see Browne, 2008b) allows for deep tactics and has also a strategic component. Most matches take less than two dozens of moves. There are almost no draws. By the way: also the game's name "Yavalath" was created by a program designed by $\mathrm{C}$. Browne.

Browne is aware of the problem that it might result in a disaster when too many new board games were brought to the market. Therefore, he did not make LUDI publicly available... For our computer game scene, C.B. is a stroke of luck. He is not only a scientist of highest calibre but also a talent with the pencil. Long before the Ph.D. project he wrote two books which have become classics: in 2000 the one on Hex (Browne, 2000), and in 2005 the bible of connection games (Browne, 2005). Also, as a traditional game designer Browne is very creative and successful, documented by (currently) 51 games listed in the BoardGameGeek online repository (Browne, visited by I.A. on December 2010).

Browne's thesis is an ideal starting point for engaged master theses: take some (large) class of games and some appropriate game search algorithm (such as Monte-Carlo based tree search for connection games or games of territory). Do all the technical homework (such as in part II of Browne's thesis) in "the right way", and at the end make a run to obtain fantastic new games.

Browne's thesis is the seminal paper of "fully automatic" board game inventing.

\section{References}

Althöfer, I. (2003). Computer-aided game inventing. Report, FSU Jena, Fakultät Mathematik und Informatik, 2003. Downloadable from http://www.minet.uni-jena.de/preprints/althoefer_03/CAGI.pdf .

Browne, C (2000). Hex Strategy: Making The Right Connections, AK Peters, Natich, Massachusetts.

Browne, C. (2005). Connection Games: Variations on a Theme, AK Peters, Natich, Massachusetts.

Browne, C. (2008a). Automatic Generation and Evaluation of Recombination Games. Ph.D. thesis, Queensland University of Technology, February 2008. Downloadable from http://www.cameronius.com/

Browne, C. (2008b). Game Yavalath. Automatically invented within his Ph.D. project. Online description at http://www.cameronius.com/ in the games section.

Browne, C. Online list of 51 games invented by him, at http://www.boardgamegeek.com/boardgamedesigner/3541/cameron-browne (visited on December 15, 2010). 